\title{
Two-State Theory of Nonlinear Stochastic Resonance
}

\author{
Jesús Casado-Pascual, José Gómez-Ordóñez, and Manuel Morillo \\ Física Teórica, Universidad de Sevilla, Apartado de Correos 1065, Sevilla 41080, Spain \\ Peter Hänggi \\ Institut für Physik, Universität Augsburg, Universitätsstraße 1, D-86135 Augsburg, Germany
}

(Received 25 July 2003; published 20 November 2003)

\begin{abstract}
An amenable, analytical two-state description of the nonlinear population dynamics of a noisy bistable system driven by a rectangular subthreshold signal is put forward. Explicit expressions for the driven population dynamics, the correlation function (its coherent and incoherent parts), the signal-tonoise ratio (SNR), and the stochastic resonance (SR) gain are obtained. Within a suitably chosen range of parameter values this reduced description yields anomalous SR gains exceeding unity and, simultaneously, gives rise to a nonmonotonic behavior of the SNR vs the noise strength. The analytical results agree well with those obtained from numerical solutions of the Langevin equation.
\end{abstract}

DOI: 10.1103/PhysRevLett.91.210601

PACS numbers: 05.40.-a, 02.50.-r, 05.10.Gg

The phenomenon of stochastic resonance (SR) attracts ever growing interest due to its multifaceted relevance for a variety of noise-induced features in physics, chemistry, and the life sciences [1-5]. Several SR quantifiers have been used to characterize the response of a noisy system to the action of time-periodic external forces. In particular, the nonmonotonic behavior of the output signal-tonoise ratio (SNR) with the strength of the noise has been used widely. A dimensionless quantity that measures the "quality" of the response with respect to the input signal is the SR gain defined as the ratio of the output SNR over the input SNR. Ideally, one would wish to obtain the characteristic amplification of the SR phenomenon $[2,6]$ and, simultaneously, SR gains larger than unity. For superthreshold sinusoidal input signals SR gains larger than unity have been reported before [7]. In recent analog [8-10] and numerical [11,12] simulations of noisy bistable systems driven by subthreshold multifrequency input forces, surprisingly large SR gains larger than unity have been established.

In order to clarify the conditions under which these anomalous large SR gains occur, it would be interesting to propose simplified models, amenable to analytical treatment, which describe this rich behavior of the response. A detailed proof that SR gains larger than unity are incompatible with linear response theory (LRT) has been presented in [11]. Thus, any theoretical explanation of the simultaneous existence of SR and anomalous large gains is rooted in the response beyond LRT.

The main focus of this Letter is to present such a simplified two-state description of the nonlinear dynamics of a noisy, symmetric bistable system driven by a rectangular subthreshold signal. A two-state description of SR has been considered previously in the pioneering work by McNamara and Wiesenfield for sinusoidal input signals [13]. In clear contrast to their work, however, we will here not linearize the transition probabilities in the strength of the applied force. In doing so, we put forward explicit analytical expressions for the driven population probabilities, the nonlinear correlation function (its coherent and incoherent part), the SNR and the SR gain. These novel nonlinear response results come forth solely because the rectangular signal-in contrast to a sinusoidal driving - involves two force values only.

To start, let us consider a system characterized by a single degree of freedom, $x$, whose dynamics (in dimensionless units) is governed by the Langevin equation

$$
\dot{x}(t)=-U^{\prime}[x(t), t]+\xi(t),
$$

where $\xi(t)$ is a Gaussian white noise of zero mean with $\langle\xi(t) \xi(s)\rangle=2 D \delta(t-s)$, and $-U^{\prime}(x, t)$ represents the force stemming from the time-dependent, archetype bistable potential

$$
U(x, t)=\frac{x^{4}}{4}-\frac{x^{2}}{2}-F(t) x .
$$

We will restrict our study to a periodic rectangular driving force with period $T$,

$$
F(t)=(-1)^{n(t)} A,
$$

where $n(t)=\lfloor 2 t / T\rfloor,\lfloor z\rfloor$ being the floor function of $z$, i.e., the greatest integer less than or equal to $z$. In other words, $F(t)=A[F(t)=-A]$ if $t \in[n T / 2,(n+1) T / 2]$ with $n$ even (odd). Our focus is on subthreshold signals; more precisely, we will assume that $A<A_{\mathrm{th}}=\sqrt{4 / 27}$ where $A_{\text {th }}$ is the static threshold value (the dynamical threshold value always exceeds this adiabatic threshold $A_{\text {th }}$ ). In this case, the potential $U(x, t)$ presents two minima at $x_{1}(t)<0$ and $x_{2}(t)>0$, and a maximum at $x_{M}(t)$. Because the potential fulfills the symmetry property $U(x, t+T / 2)=U(-x, t)$, then $x_{M}(t)=(-1)^{n(t)} x_{M}(0)$, and 


$$
x_{j}(t)=(-1)^{j} \frac{\Delta x(0)}{2}-(-1)^{n(t)} \frac{x_{M}(0)}{2},
$$

where $\Delta x(0)=x_{2}(0)-x_{1}(0)$. Additionally, we have taken into account the symmetry relation $x_{1}(t)+x_{2}(t)+$ $x_{M}(t)=0$.

Throughout the following, we will assume that the noise strength, $D$, is sufficiently small so that the intrawell relaxation time scale is negligible compared with the time scale associated to the interwell transitions and, as well, the driving time scale $T / 2$. In this case, and for sufficiently slow driving [14,15], the exact Langevin dynamics can be approximated by a nonstationary, Markovian two-state description of the form

$$
\begin{aligned}
& \dot{P}(1, t)=-\gamma_{1}(t) P(1, t)+\gamma_{2}(t) P(2, t), \\
& \dot{P}(2, t)=-\gamma_{2}(t) P(2, t)+\gamma_{1}(t) P(1, t) .
\end{aligned}
$$

Here, $P(1, t)$ and $P(2, t)$ denote, respectively, the populations to the left and to the right of the maximum position, $x_{M}(t)$, and $\gamma_{j}(t)$ is the Kramers rate of escape [16] from the well $j$ at time $t$, i.e.,

$$
\gamma_{j}(t)=\frac{\omega_{j}(t) \omega_{M}(t)}{2 \pi} \exp \left\{-\frac{U\left[x_{M}(t), t\right]-U\left[x_{j}(t), t\right]}{D}\right\},
$$

where $\quad \omega_{j}(t)=\sqrt{U^{\prime \prime}\left[x_{j}(t), t\right]}=\sqrt{3\left[x_{j}(t)\right]^{2}-1} \quad$ and $\omega_{M}(t)=\sqrt{\left|U^{\prime \prime}\left[x_{M}(t), t\right]\right|}=\sqrt{1-3\left[x_{M}(t)\right]^{2}}=\omega_{M}(0)$. According to the above mentioned symmetry property of the potential, $\gamma_{j}(t)$ can be expressed in the form

$$
\gamma_{j}(t)=\frac{\Gamma}{2}\left[1-(-1)^{n(t)+j} \Delta P_{\mathrm{eq}}(0)\right],
$$

where $\quad \Gamma=\gamma_{1}(0)+\gamma_{2}(0)=\gamma_{1}(t)+\gamma_{2}(t), \quad$ and $\Delta P_{\text {eq }}(0)=P_{\text {eq }}(2,0)-P_{\text {eq }}(1,0), P_{\text {eq }}(j, 0)$ being the equilibrium population of the state $j$ corresponding to the rates taken at time $t=0$, i.e., $P_{\text {eq }}(j, 0)=\left[\delta_{j, 1} \gamma_{2}(0)+\right.$ $\left.\delta_{j, 2} \gamma_{1}(0)\right] / \Gamma$. Equations (5) and (6) describe the evolution of the population between two consecutive changes of shape of the potential. These populations can be discontinuous at $t=n T / 2$, as a result of the sudden change in the location of the maximum at those instants of time. Therefore, in order to complete the description, jump conditions for the populations at $t=n T / 2$ have to be added. For our situation with a rectangular signal we shall assume that the probability distribution before a change of the potential is sufficiently well localized around the minima that there is almost no probability transfer from one well to the other as the maximum location changes. In this case, the populations can be considered to be continuous at $t=n T / 2$, i.e.,

$$
\lim _{t \rightarrow(n T / 2)-} P(j, t)=\lim _{t \rightarrow(n T / 2)+} P(j, t)=P(j, n T / 2) .
$$

This relation becomes exact at asymptotic weak noise. At finite weak noise strength $D$ those finite jump conditions induce for the correlation function some small, weakly nonanalytic structures which in turn may cause diplike features around even numbered multiples of the angular driving frequency $\Omega$. Indeed, such dips in the spectrum are well known to occur at weak noise for timecontinuous, e.g., sinusoidally rocked bistable systems in the nonlinear response regime [17]; note, however, that for our case with a constant force (or potential) the source of the nonanalytic structure is of different origin. For rectangular driving forces and noise strength values leading to SR gains larger than unity, the incoherent part of the correlation decays very rapidly on the time scale $T / 2$ so that these small corrections stemming from the approximation in (9) can safely be neglected. After using the normalization condition $P(1, t)+P(2, t)=1$, Eqs. (5) and (6) yield

$$
\dot{P}(1, t)=-\Gamma P(1, t)+\gamma_{2}(t) .
$$

Upon observing that $\gamma_{2}(t)$ remains constant between two consecutive changes of the potential, the solution of Eq. (10) can be expressed as

$$
P(1, t)=\frac{\gamma_{2}(t)}{\Gamma}+\left\{P[1, n(t) T / 2]-\frac{\gamma_{2}(t)}{\Gamma}\right\} e^{-\Gamma[t-(n(t) T / 2)]} .
$$

With this relation, it is straightforward to evaluate the time-periodic, asymptotic longtime solution of Eq. (10), i.e., $P_{\infty}(1, t)$. In order to do so, we calculate the values $P_{\infty}(1, n T / 2)$ by making use of the symmetry property $P_{\infty}(1, t \pm T / 2)=P_{\infty}(2, t)=1-P_{\infty}(1, t)$, as well as of Eq. (9). After inserting the result in Eq. (11), one finds the $T$-periodic solution

$$
\begin{aligned}
P_{\infty}(1, t)= & \frac{1}{2}\left[1-(-1)^{n(t)} \Delta P_{\mathrm{eq}}(0)\right] \\
& +(-1)^{n(t)} \Delta P_{\mathrm{eq}}(0) \frac{e^{-\Gamma[t-(n(t) T / 2)]}}{1+e^{-(\Gamma T / 2)}} .
\end{aligned}
$$

The average of the coordinate in the longtime limit, $\langle x(t)\rangle_{\infty}^{(T S)}=\sum_{j=1}^{2} x_{j}(t) P_{\infty}(j, t)$, is evaluated to read

$$
\begin{aligned}
\langle x(t)\rangle_{\infty}^{(T S)}=(-1)^{n(t)} & \left\{\langle x(0)\rangle_{\mathrm{eq}}-\left[x_{M}(0)+2\langle x(0)\rangle_{\mathrm{eq}}\right]\right. \\
& \left.\times \frac{e^{-\Gamma[t-(n(t) T / 2)]}}{1+e^{-(\Gamma T / 2)}}\right\},
\end{aligned}
$$

where $\langle x(0)\rangle_{\mathrm{eq}}=\sum_{j=1}^{2} x_{j}(0) P_{\mathrm{eq}}(j, 0)$.

The conditional probability $P\left(1, t \mid j, t_{0}\right)$ can also be calculated using the observation that $P\left(1, t \mid j, t_{0}\right)-$ $P_{\infty}(1, t)$ (for $t \geq t_{0}$ ) fulfills the homogeneous equation obtained by removing the term $\gamma_{2}(t)$ from Eq. (10), with the initial condition $P\left(1, t_{0} \mid j, t_{0}\right)-P_{\infty}\left(1, t_{0}\right)=$ $\delta_{j, 1}-P_{\infty}\left(1, t_{0}\right)$. Thus, the result for $t \geq t_{0}$ is

$$
P\left(1, t \mid j, t_{0}\right)=P_{\infty}(1, t)+\left[\delta_{j, 1}-P_{\infty}\left(1, t_{0}\right)\right] e^{-\Gamma\left(t-t_{0}\right)},
$$


and, likewise, $P\left(2, t \mid j, t_{0}\right)=1-P\left(1, t \mid j, t_{0}\right)$. In terms of the time-periodic one-time probability in Eq. (12) the two-time joint probability reads

$$
\begin{aligned}
P_{\infty}\left(j, t ; k, t_{0}\right)= & (-1)^{j+k} P_{\infty}\left(1, t_{0}\right) P_{\infty}\left(2, t_{0}\right) e^{-\Gamma\left(t-t_{0}\right)} \\
& +P_{\infty}(j, t) P_{\infty}\left(k, t_{0}\right),
\end{aligned}
$$

for $t \geq t_{0}$. Therefore, the asymptotic two-time correlation function, $\left\langle x(t) x\left(t_{0}\right)\right\rangle_{\infty}^{(T S)}=\sum_{j=1}^{2} \sum_{k=1}^{2} x_{j}(t) x_{k}\left(t_{0}\right) \times$ $P_{\infty}\left(j, t ; k, t_{0}\right)$, reads

$$
\begin{aligned}
\left\langle x(t) x\left(t_{0}\right)\right\rangle_{\infty}^{(T S)}= & {[\Delta x(0)]^{2} P_{\infty}\left(1, t_{0}\right) P_{\infty}\left(2, t_{0}\right) e^{-\Gamma\left(t-t_{0}\right)} } \\
& +\langle x(t)\rangle_{\infty}^{(T S)}\left\langle x\left(t_{0}\right)\right\rangle_{\infty}^{(T S)},
\end{aligned}
$$

for $t \geq t_{0}$, where we have used that $\Delta x(t)=\Delta x(0)$.

The two-time correlation function $\left\langle x\left(t_{0}+\tau\right) x\left(t_{0}\right)\right\rangle_{\infty}^{(T S)}$ is a periodic function of $t_{0}$ with the period of the external driving [6]. Then, it is convenient to apply a time average to obtain the time-homogenous correlation function, $C^{(T S)}(\tau)$, i.e.,

$$
C^{(T S)}(\tau)=\frac{1}{T} \int_{0}^{T} d t_{0}\left\langle x\left(t_{0}+\tau\right) x\left(t_{0}\right)\right\rangle_{\infty}^{(T S)} .
$$

In virtue of Eq. (16), $C^{(T S)}(\tau)$ can be written as the sum of two contributions: a coherent part, $C_{\mathrm{coh}}^{(T S)}(\tau)$, which is periodic in $\tau$ with period $T$, and an incoherent part, $C_{\text {incoh }}^{(T S)}(\tau)$, which decays to 0 as $\tau \rightarrow \infty$. These two contributions are given by, respectively,

$$
C_{\mathrm{coh}}^{(T S)}(\tau)=\frac{1}{T} \int_{0}^{T} d t_{0}\left\langle x\left(t_{0}+\tau\right)\right\rangle_{\infty}^{(T S)}\left\langle x\left(t_{0}\right)\right\rangle_{\infty}^{(T S)},
$$

and

$$
C_{\text {incoh }}^{(T S)}(\tau)=\frac{[\Delta x(0)]^{2} e^{-\Gamma \tau}}{T} \int_{0}^{T} d t_{0} P_{\infty}\left(1, t_{0}\right) P_{\infty}\left(2, t_{0}\right) .
$$

Upon combining Eqs. (12), (13), (18), and (19), one obtains after some cumbersome algebra

$$
C_{\mathrm{coh}}^{(T S)}(\tau)=(-1)^{n(t)}\left\{\langle x(0)\rangle_{\mathrm{eq}}^{2}\left[2 n(\tau)+1-\frac{4 \tau}{T}\right]+\frac{4 \operatorname{sech}\left(\frac{\Gamma T}{4}\right) \sinh \left\{\frac{\Gamma T}{4}\left[2 n(\tau)+1-\frac{4 \tau}{T}\right]\right\}}{\Gamma T}\left[\left[\frac{x_{M}(0)}{2}\right]^{2}-\langle x(0)\rangle_{\mathrm{eq}}^{2}\right]\right\},
$$

and

$$
\begin{aligned}
& C_{\text {incoh }}^{(T S)}(\tau)=\frac{[\Delta x(0)]^{2} e^{-\Gamma \tau}}{4}\{1+\left[\Delta P_{\mathrm{eq}}(0)\right]^{2} \\
&\left.\times\left[\frac{4 \tanh \left(\frac{\Gamma T}{4}\right)}{\Gamma T}-1\right]\right\} .
\end{aligned}
$$

According to McNamara and Wiesenfeld [13], the output $\mathrm{SNR}, R_{\text {out }}^{(T S)}$, is defined in terms of the Fourier transform of the coherent and incoherent parts of $C^{(T S)}(\tau)$ as

$$
R_{\text {out }}^{(T S)}=\frac{\lim _{\epsilon \rightarrow 0+} \int_{\Omega-\epsilon}^{\Omega+\epsilon} d \omega \tilde{C}^{(T S)}(\omega)}{\tilde{C}_{\text {incoh }}^{(T S)}(\Omega)},
$$

where $\Omega=2 \pi / T$ is the angular frequency of the external driving, and $\tilde{H}(\omega)$ denotes the Fourier cosine transform of $H(\tau)$, i.e., $\tilde{H}(\omega)=2 / \pi \int_{0}^{\infty} d \tau H(\tau) \cos (\omega \tau)$. Note that this definition of the output SNR differs by a factor 2 , stemming from the same contribution at $\omega=-\Omega$, from the definitions used in earlier works $[2,6]$. The periodicity of the coherent part gives rise to delta peaks in the spectrum. Thus, the only contribution to the numerator in Eq. (22) stems from the coherent part of the correlation function. The output SNR can then be expressed as

$$
R_{\mathrm{out}}^{(T S)}=\frac{Q_{u}^{(T S)}}{Q_{l}^{(T S)}},
$$

where

$$
Q_{u}^{(T S)}=\frac{2}{T} \int_{0}^{T} d \tau C_{\mathrm{coh}}^{(T S)}(\tau) \cos (\Omega \tau)
$$

and

$$
Q_{l}^{(T S)}=\frac{2}{\pi} \int_{0}^{\infty} d \tau C_{\text {incoh }}^{(T S)}(\tau) \cos (\Omega \tau)
$$

Then, from Eqs. (20), (21), (24), and (25), we get after some simplifications

$$
Q_{u}^{(T S)}=\frac{2\left[4\langle x(0)\rangle_{\mathrm{eq}}^{2} \Gamma^{2}+\left[x_{M}(0)\right]^{2} \Omega^{2}\right]}{\pi^{2}\left(\Gamma^{2}+\Omega^{2}\right)},
$$

and

$$
\begin{aligned}
Q_{l}^{(T S)}=\frac{[\Delta x(0)]^{2} \Gamma}{2 \pi\left(\Gamma^{2}+\Omega^{2}\right)}\{1 & +\left[\Delta P_{\mathrm{eq}}(0)\right]^{2} \\
& \left.\times\left[\frac{4 \tanh \left(\frac{\Gamma T}{4}\right)}{\Gamma T}-1\right]\right\} .
\end{aligned}
$$

The signal-to-noise ratio of the input signal, $F(t)+\xi(t)$, can readily be evaluated from the definition, yielding

$$
R_{\text {inp }}=\frac{4 A^{2}}{\pi D} \text {. }
$$

Thus, the SR gain which is defined as the ratio of the SNR of the output over the SNR of the input, emerges as

$$
G^{(T S)}=\frac{R_{\text {out }}^{(T S)}}{R_{\text {inp }}},
$$

and it can be evaluated explicitly upon combining the set of Eqs. (23) and (26)-(28).

In Fig. 1 we compare our analytical results for the behavior of several SR quantifiers as a function of the noise strength $D$ with results obtained by numerically integrating the Langevin equation [Eq. (1)]. The 

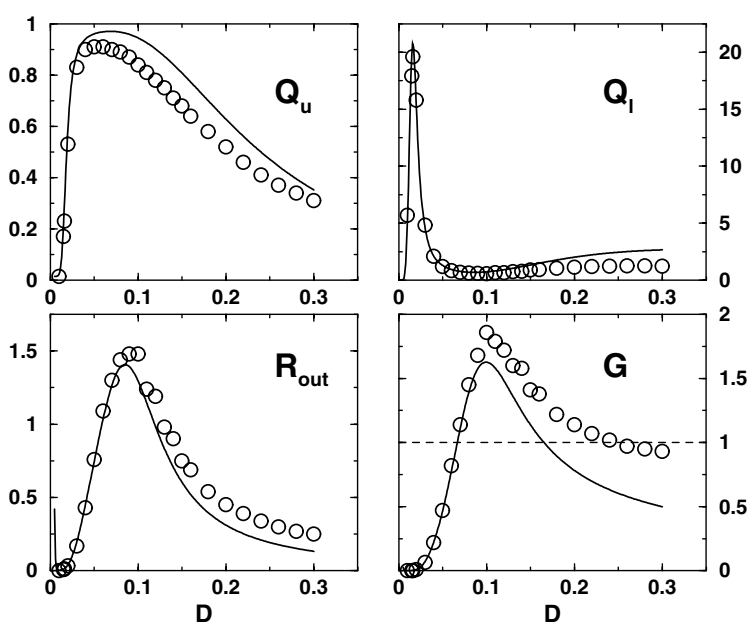

FIG. 1. Stochastic resonance beyond linear response: Several SR quantifiers are depicted vs the noise strength $D$; namely, the cosine transform of the coherent part of the driven correlation function at angular driving frequency $\Omega$, i.e., the numerator of the $\operatorname{SNR}\left(Q_{u}\right)$, and the cosine transform of the incoherent part, i.e., the denominator $\left(Q_{l}\right)$, the output SNR $\left(R_{\text {out }}\right)$ and the SR gain $(G)$. These characteristic quantities are evaluated for a rectangular driving force with angular frequency $\Omega=0.01$ and subthreshold amplitude $A=0.25$. The solid lines depict the results obtained within the two-state description, whereas the numerical precise results obtained from the driven Langevin dynamics in Eqs. (1)-(3) are given by the circles.

numerical precise solution is based on an algorithm due to Greenside and Helfand; for details see in the Appendix of Ref. [11]. The value of the subthreshold input amplitude is chosen as $A=0.25$, and the frequency of the external driving is set at $\Omega=0.01$. This small value of the angular frequency has been chosen in order to observe a characteristic nonmonotonic behavior versus $D$ of several quantifiers associated to SR and, simultaneously, SR gains exceeding unity over a wide range of $D$ (see Ref. [12]). The agreement between the analytical nonlinear response results and the numerical results is surprisingly good at moderate noise values, and becomes even excellent for small values of $D$. The better agreement at low noise values corroborates the fact that with increasing noise strength the Markovian two-state description also worsens [15].

With this Letter we have put forward an analytical two-state description for both the nontrivial, nonlinear population dynamics and the nonstationary correlation behavior of noisy bistable systems driven by periodic rectangular subthreshold forces. It is indeed remarkable that our analytical, nonlinear two-state approach does capture well both the nonmonotonic, bell-shaped behavior of the nonlinear SNR vs noise strength $D$, i.e., the characteristic SR phenomenon, and the occurrence of SR gains larger than unity. This latter result is a true benchmark of the nonlinear response behavior of a driven bistable stochastic dynamics. Our analytical findings cor- roborate those obtained by means of numerical solutions of the Langevin equation [12].

This very two-state theory beyond linear response of a driven, metastable stochastic population dynamics likely proves useful also for phenomena other than stochastic resonance: it equally well can be applied and generalized to describe the behavior of rocked Brownian motors [18], the description of the switching dynamics over adiabatically sloshing potential landscapes [19] or also to driven noisy threshold characteristics in general, such as, e.g., for a driven neuronal noisy spiking dynamics [20].

The authors acknowledge the support of the Dirección General de Enseñanza Superior of Spain (BFM200203822), the Junta de Andalucía, DAAD-Acciones Integradas (P. H., M. M.), and the Sonderforschungsbereich 486 of the DFG.

[1] A. R. Bulsara and L. Gammaitoni, Phys. Today 49, No. 3, 39 (1996).

[2] L. Gammaitoni, P. Hänggi, P. Jung, and F. Marchesoni, Rev. Mod. Phys. 70, 223 (1998).

[3] K. Wiesenfeld and F. Jaramillo, Chaos 8, 539 (1998).

[4] V.S. Anishchenko, A. B. Neiman, F. Moss, and L. Schimansky-Geier, Usp. Fiz. Nauk 169, 7 (1999) [Phys. Usp. 42, 7 (1999)].

[5] P. Hänggi, Chem. Phys. Chem. 3, 285 (2002).

[6] P. Jung and P. Hänggi, Phys. Rev. A 44, 8032 (1991).

[7] P. Hänggi, M. Inchiosa, D. Fogliatti, and A. R. Bulsara, Phys. Rev. E 62, 6155 (2000).

[8] K. Loerincz, Z. Gingl, and L. B. Kiss, Phys. Lett. A 224, 63 (1996).

[9] Z. Gingl, R. Vajtai, and P. Makra, in ICNF 2001, edited by G. Bosman (World Scientific, Singapore, 2002), pp. 545-548.

[10] Z. Gingl, P. Makra, and R. Vajtai, Fluct. Noise Lett. 1, L181 (2001).

[11] J. Casado-Pascual et al., Phys. Rev. E 67, 036109 (2003).

[12] J. Casado-Pascual et al., cond-mat/0307589 [Phys. Rev. E (to be published)].

[13] B. McNamara and K. Wiesenfeld, Phys. Rev. A 39, 4854 (1989).

[14] I. Derenyi and R. D. Astumian, Phys. Rev. Lett. 82, 2623 (1999); M. Bier, et al., Phys. Rev. E 59, 6422 (1999).

[15] P. Talkner and J. Luczka, cond-mat/0307498.

[16] P. Hänggi, P. Talkner, and M. Borkovec, Rev. Mod. Phys. 62, 251 (1990).

[17] V. A. Shneidman, P. Jung, and P. Hänggi, Europhys. Lett. 26, 571 (1994); V. A. Shneidman et al., Phys. Rev. Lett. 72, 2682 (1994).

[18] R. D. Astumian and P. Hänggi, Phys. Today 55, No. 11, 33 (2002); P. Reimann, Phys. Rep. 361, 57 (2002).

[19] J. Lehmann, P. Reimann, and P. Hänggi, Phys. Rev. Lett. 84, 1639 (2000); R. S. Maier and D. L. Stein, Phys. Rev. Lett. 86, 3942 (2001); J. Lehmann et al., Phys. Status Solidi (b) 237, 53 (2003).

[20] I. A. Khovanov and P.V. E. McClintock, Phys. Rev. E 67, 043901 (2003). 